Research Article

\title{
Identification and characterization of MAGO and $Y 14$ genes in Hevea brasiliensis
}

\author{
Zi-Ping Yang ${ }^{1,2}$, Hui-Liang $\mathrm{Li}^{1}$, Dong Guo ${ }^{1}$ and Shi-Qing Peng ${ }^{1,2, \#}$ \\ ${ }^{1}$ Key Laboratory of Biology and Genetic Resources of Tropical Crops, Ministry of Agriculture, Institute of \\ Tropical Bioscience and Biotechnology, Chinese Academy of Tropical Agricultural Sciences, Haikou, China \\ ${ }^{2}$ College of Agriculture, Hainan University, Haikou, China
}

\begin{abstract}
Mago nashi (MAGO) and Y14 proteins are highly conserved among eukaryotes. In this study, we identified two MAGO (designated as HbMAGO1 and HbMAGO2) and two Y14 (designated as HbY14a and HbY14b) genes in the rubber tree (Hevea brasiliensis) genome annotation. Multiple amino acid sequence alignments predicted that $\mathrm{HbMAGO}$ and $\mathrm{HbY} 14$ proteins are structurally similar to homologous proteins from other species. Tissue-specific expression profiles showed that HbMAGO and HbY14 genes were expressed in at least one of the tissues (bark, flower, latex, leaf and root) examined. HbMAGOs and HbY14s were predominately located in the nucleus and were found to interact in yeast two-hybrid analysis (YTH) and bimolecular fluorescence complementation (BiFC) assays. $\mathrm{HbMAGOs}$ and $\mathrm{HbY} 14 \mathrm{~s}$ showed the highest transcription in latex and were regulated by ethylene and jasmonate. Interaction between HbMAGO2 and $\mathrm{gp} 91^{\text {phox }}$ (a large subunit of nicotinamide adenine dinucleotide phosphate) was identified using YTH and BiFC assays. These findings suggested that HbMAGO may be involved in the aggregation of rubber particles in $H$. brasiliensis.
\end{abstract}

Keywords: interaction, Hevea brasiliensis, Mago nashi, Y14 proteins

Received: December 24, 2014; Accepted: June 08, 2015.

\section{Introduction}

The exon junction complex (EJC) regulates posttranscriptional events that include mRNA intracellular export, cytoplasmic localization, non-sense mediated mRNA decay (NMD) and translation enhancement in metazoans (Park and Muench, 2007; Lee et al., 2009; Ashton-Beaucage et al., 2010; Roignant and Treisman, 2010; Boothby and Wolniak, 2011; Mufarrege et al., 2011). The EJC is a multiprotein complex assembled onto mRNAs as a consequence of splicing and is thought to provide a molecular link between splicing and post-splicing mRNA metabolism 20-24 bases upstream of mRNA exon-exon junctions (Le et al., 2000, 2003; Tange et al., 2004; Gehring et al., 2009). Several of the EJC components have been identified and classified into two groups, namely, EJC "core" and "peripheral" factors. Four proteins form the EJC core: MAGO (short form of MAGO NASHI and known as MAGOH in humans), Y14 (also known as Tsunagi or RBM8A), eIF4A/eIF4A3 and BTZ (short form of Barentsz, also

Send correspondence to Shi-Qing Peng. Key Laboratory of Biology and Genetic Resources of Tropical Crops, Ministry of Agriculture, Institute of Tropical Bioscience and Biotechnology, Chinese Academy of Tropical Agricultural Sciences, Haikou 571101, China. E-mail: shqpeng@163.com

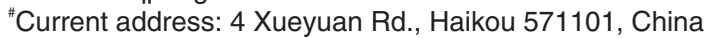

known as MLN51), of which MAGO and Y14 constitute a stable heterodimeric complex in vitro and in vivo (Tange et al., 2004, 2005; Le and Seraphin, 2008). Additional components of the EJC include the splicing factors SRm160, Pinin and RnpS1, the NMD factors Upf1, Upf2 and Upf3, the translation factors Skar and Pym, and the export factors UAP56 and REF/Aly (Roignant and Treisman, 2010). These proteins are considered to be peripheral EJC components that are transiently associated with the EJC core in the nucleus or cytoplasm, where they are involved in splicing, export, translation and NMD (Lejeune and Maquat, 2005; Tange et al., 2005).

The MAGO (meaning "no grandchildren" in Japanese) gene was first identified as a strict maternal effect gene in Drosophila (Boswell et al., 1991; Newmark and Boswell, 1994; Micklem et al., 1997; Newmark et al., 1997). MAGO can interact with an RNA binding protein, $\mathrm{Y} 14$, to regulate cell growth and is expressed throughout the organism (Zhao et al., 1998, 2000; Le et al., 2001; Mohr et al., 2001; Chen et al., 2007). Based on the crystal structures of the Drosophila and human MAGO-Y14 complex, MAGO and Y14 proteins are core components of the EJC and can form a stable heterodimer that strongly associates with spliced mRNA (Lau et al., 2003; Shi and Xu, 2003). Both MAGO and Y14 are evolutionarily highly conserved 
proteins (Hachet and Ephrussi, 2001; Mohr et al., 2001) and slow co-evolution of the two protein families is required for the maintenance of their obligate heterodimerization mode (Gong et al., 2014). Comparative sequence analysis reveals that MAGO is devoid of known structural motifs while Y14 contains a central RNA-binding domain (Kataoka et al., 2000; Kim et al., 2001; Shi and Xu, 2003). The crystal structure of the heterodimeric complexes, formed in the absence of RNA, shows that the RNAbinding motif is used for Y14/MAGO protein-protein interaction and is not readily available for binding RNA (Fribourg et al., 2003; Lau et al., 2003; Shi and Xu, 2003).

In plants, MAGO proteins have been studied to varying degrees (Chen et al., 2007; Gong and He, 2014). The orthologs of MAGO genes are linked to male fertility. For example, PFMAGO proteins interact with MADS-domain protein MPF2 and are responsible for male fertility and calyx development in Physalis (He et al., 2007). In Arabidopsis, AtMAGO is required for pollen grain development (Johnson et al., 2004) and embryo development, as well as meristem cell death events (Park et al., 2009). MAGO is essential for spermatogenesis as shown by RNAi knockdown of MvMAGO in Marsilea (van der Weele et al., 2007; Boothby and Wolniak, 2011). MAGO genes are also involved in the development of other organs, such as leaves, stems, roots and seeds. The overexpression of $T c M A G O$ in transgenic tobacco plants results in longer roots and a more complex root system (Chen et al., 2007). In Arabidopsis, RNAi-AtMAGO plants produce a greater number of leaves, short and fasciated stems, short lateral roots and non-viable seeds (Park et al., 2009). OsMAGO knockdown generated short rice plants with abnormal flowers (Gong and He, 2014). The function of MAGO as a component of the EJC is well-understood, and some functions of MAGO have been reported for other plants; however, the existence of a duplicated MAGO gene in rubber trees is not widely known.

The rubber tree (Hevea brasiliensis) is the main renewable world-wide source of commercial natural rubber (NR), a biopolymer of high economic interest composed mainly of cis-1,4-polyisoprene. Latex is produced by laticifers after tapping and is a white cytoplasmic colloidal suspension containing mainly rubber particles, but also nonrubber particles, organelles, proteins and serum. In this study, the MAGO and Y14 gene families in rubber trees were characterized. To investigate their evolutionary relationships and functions, we undertook structural and phylogenetic analyses and examined the subcellular locations of these proteins. The expression profiles of MAGOs and Y14s in various organs and the response to ethylene (ET) and jasmonate (JA) were also studied. Furthermore, the interaction partners of HbMAGO were identified in latex and the HbMAGO2 interaction with gp9 $1^{\text {phox }}$ (a large subunit of nicotinamide adenine dinucleotide phosphate) was demonstrated using yeast two-hybrid analysis (YTH) and bimo- lecular fluorescence complementation (BiFC) assays. Our results indicate that HbMAGO may be involved in the aggregation of natural rubber in rubber trees.

\section{Materials and Methods}

\section{Plant materials}

Hevea brasiliensis cultivar RY7-33-97 was planted on the experimental farm of the Chinese Academy of Tropical Agriculture Sciences, Hainan, China. The shoots were treated with $0.5 \%$ ethylene (ET) or $0.1 \%$ jasmonic acid (JA), as described by Hao and Wu (2000). Latex samples were collected 1, 3, 6, 9, 24 and $48 \mathrm{~h}$ after treatment from 12 shoots for each interval and were immediately stored at $-80{ }^{\circ} \mathrm{C}$ for RNA extraction. Three independent biological replicates were done for each treatment. For latex RNA extraction, the latex was dropped directly into liquid nitrogen in an ice kettle. Rubber tree flowers, leaves and bark were washed with double-distilled water to remove latex and then immediately frozen in liquid nitrogen.

\section{Database search and sequence conservation analysis of rubber tree MAGO and Y14 genes}

A local whole genome shotgun database for rubber tree was established by Yang et al. (2014). The full-length cDNA of HbMAGO2 was obtained from a yeast twohybrid cDNA library of $H$. brasiliensis laticifers using HbWRKY as the bait in the yeast two-hybrid assay (data unpublished). HbY14a was obtained from the yeast twohybrid cDNA library of $H$. brasiliensis laticifers using $\mathrm{HbMAGO} 2$ as the bait. HbMAGO2 and HbY14a were used as queries in Blast searches of the rubber tree whole genome shotgun database. The shotgun sequences that produced the highest significant alignments judged by the scores and E-values were used for HMM-based gene structure prediction (http://linux1.softberry.com/berry.phtml) based on the Arabidopsis and rubber tree databases. The genomic DNA and cDNA sequences of four genes were amplified using appropriate primers (developed based on gene structure prediction) and sequenced.

\section{Phylogenetic analysis and genomic structure}

Analysis and comparison of the HbMAGO and HbY14 sequences were done using BLAST at the NCBI database. Clustal X (http://www.ebi.ac.uk/clustalw) was used for multiple alignments of the nucleic acid and amino acid sequences of HbMAGOs and HbY14s. A phylogenetic tree was constructed with MEGA5.2 (http://www.megasoftware.net/) using the maximum likelihood (ML) method with a bootstrap parameter of 1000 replicates. The sequence information for a number of homologs from other species was retrieved from the NCBI database and is shown in the explanatory text of Figure 2. Genomic structures of the HbMAGOs and HbY14s were analyzed by comparing the cDNA sequences using GSDS 
software (http://gsds.cbi.pku.edu.cn/) and the corresponding genomic DNA sequences that were extracted from the local whole genome shotgun database.

\section{Transient protein expression}

To determine the subcellular location of the proteins, full-length cDNAs were cloned (see Table 1 for primers) and inserted into the vector pCAMBIAC1302 driven by the $35 \mathrm{~S}$ promoter. The resulting pCAMBIA1302 constructs were first transformed into the GV3101 strain of Agrobacterium tumefaciens by electroporation (Gene Pulser Xcell, Bio-Rad, USA) and then introduced into onion epidermal cells by agroinfiltration using a water circulating vacuum pump (model SHZ-III B, Shanghai, China) (Yang et al., 2000, 2014). The empty GFP vector was infiltrated as a control. For the BiFC assay, the open reading frames of HbMAGO2 and gp91 $91^{\text {phox }}$ (without their stop codons) were subcloned into pSPYNE-35S (split YFP N-terminal fragment expression) or pSPYCE-35S (split YFP C-terminal fragment expression) vectors driven by the $35 \mathrm{~S}$ promoter (see Table 1 for primers). The resulting pSPYNE and pSPYCE plasmids were transformed into the GV3101 strain and the YNE- and YCE-fused proteins were then co-expressed in onion epidermal cells by agroinfiltration (Walter et al., 2004; Yang et al., 2014). Co-expressions of target genes and YFPN or YFPC were used as negative controls. The cells were dipped in $30 \%$ sucrose solution for $30 \mathrm{~min}$ to induce plasmolysis and the green fluorescent protein (GFP) or yellow fluorescent protein (YFP) signal was then examined with a confocal laser scanning microscope (Zeiss LSM510, Germany).

\section{Expression analysis}

Total RNA was extracted as described by Tang et al. (2007). First-strand cDNA was synthesized using a RevertAid $^{\mathrm{TM}}$ first-strand cDNA synthesis kit (Fermentas, Lithuania). qRT-PCR was done using the primers indicated in Table 1, with the rubber tree actin gene (GenBank HQ260674.1) being used as a reference gene. The qPCR fragments were amplified and sequenced to determine primer specificity. qRT-PCR was done using the fluorescent dye SYBR-Green (Takara, China) and the melting curves of the amplification products were assessed using a Stratagene Mx3005P real-time thermal cycler (Agilent, America). The qRT-PCR conditions were as follows: $30 \mathrm{~s}$ at $95^{\circ} \mathrm{C}$ for denaturation followed by 40 cycles of $5 \mathrm{~s}$ at 94 ${ }^{\circ} \mathrm{C}, 20 \mathrm{~s}$ at $56^{\circ} \mathrm{C}$ and $20 \mathrm{~s}$ at $72{ }^{\circ} \mathrm{C}$ for amplification. An average of three independent biological replicates was run for each time interval. The experimental results were reported as the mean \pm SEM of three repli-cates. The data were analyzed with one-way ANOVA and the level of significance was set at $p<0.01$ or $p<0.05$ (Fisher's protected least significant difference). All data analyses were done using Statistical Product and Service Solutions software (SPSS, version 16.0). 


\section{Yeast two-hybrid assays}

For yeast mating, the open reading frame of HbMAGO2 was inserted into pGBKT7 vectors to create a bait plasmid. The bait plasmid (pGBKT7-HbMAGO2) was transformed into the yeast strain AH109 by the lithium acetate method, according to the Yeastmaker yeast transformation system 2 user manual (Clontech); the yeast cells were subsequently grown on SD/-Trp medium for 3 days. The bait strain was co-incubated with the library strain to promote fusion in 2YPDA liquid medium containing kanamycin $(50 \mu \mathrm{g} / \mathrm{mL})$ and the intermixture was shaken slowly at $30{ }^{\circ} \mathrm{C}$. If zygotes were present, the mated culture was centrifuged and resuspended in 0.5YPDA/Kan liquid medium followed by plating on $\mathrm{DDO} / \mathrm{X} / \mathrm{A}$ (double dropout medium: SD/-Trp/-Leu with $\mathrm{X}-\alpha-\mathrm{Gal}$ and aureobasidin A) medium and incubation at $30{ }^{\circ} \mathrm{C}$ for 3-5 days. Plasmids were rescued from yeast, transformed into $E$. coli and the DNA then purified. The interaction was confirmed by cotransforming the library plasmid and bait into the yeast strain AH109. The cDNA inserts were subsequently sequenced to confirm their identity.

To confirm one-to-one interaction, the open reading frame of HbMAGOs, HbY14s and gp91 ${ }^{\text {phox }}$ were inserted into pGBKT7 or pGADT7 vectors to create bait or prey (primers listed in Table 1). The indicated combination of the bait and prey plasmids was co-transformed into yeast strain AH109 and the yeast cells were plated onto $\mathrm{QDO} / \mathrm{X} / \mathrm{A}$ medium (quadruple dropout medium: $\mathrm{SD} /$-Trp/-Leu/-Ade/-His with $\mathrm{X}-\alpha$-Gal and aureobasidin A). $\beta$-Galactosidase activity was measured with o-nitrophenyl- $\beta$-D-galactopyranoside (ONPG) as the substrate according to the Yeast Protocols Handbook (no. PT3024-1, Clontech). The changes in absorbance were monitored at $\mathrm{OD}_{420 \mathrm{~nm}}$. The experimental results were reported as the means \pm SEM of three repli-cates. The data were analyzed with one-way ANOVA and the level of significance was set at $\mathrm{p}<0.01$ or $\mathrm{p}<0.05$ (Fisher's protected least significant difference). All data analyses were done using Statistical Product and Service Solutions software (SPSS, version 16.0).

\section{Results}

Identification and sequence conservation of rubber tree MAGO and $\mathrm{Y} 14$ genes

Two MAGO genes (designated as HbMAGO1 and HbMAGO2) and two Y14 genes (designated as HbY14a and $H b Y 14 b)$ were identified in rubber tree. The HbMAGO1/2 proteins had calculated molecular masses of 17.476 and $17.688 \mathrm{kDa}$ and isoelectric points (pI) of 5.68 and 5.69 (Table 2). Alignment analysis indicated significant similarities among homologous proteins. The most striking feature of MAGO proteins was their highly conserved primary amino acid sequences (Figure 1A). The
HbMAGO1 protein shared 94\%, 93\%, 92\% and 92\% amino acid identity with the homologs TcMAGO, PtMAGO, CsMAGO and VvMAGO, respectively; the HbMAGO2 protein shared $94 \%, 91 \%, 92 \%$ and $91 \%$ amino acid identity with the homologs of TcMAGO, PtMAGO, CsMAGO and VvMAGO, respectively. The secondary structure prediction of the MAGO protein revealed that it consisted of six $\beta$-strands $(\beta 1-\beta 6)$ and three $\alpha$-helices $(\alpha 1-\alpha 3)$ in a $\beta 1-\beta 2-\beta 3-\beta 4-\alpha 1-\beta 5-\beta 6-\alpha 2-\alpha 3$ arrangement (Figure 1A). The $\mathrm{HbY} 14 \mathrm{a} / \mathrm{b}$ proteins had calculated molecular masses of $21.737 \mathrm{kDa}$ and $21.903 \mathrm{kDa}$ and isoelectric points (pI) of 4.79 and 4.79 (Table 2).

The HbY14a protein shared 92\%, 80\%, 72\%, 77\%, $68 \%$ and $72 \%$ amino acid identity with the homologs of RcMAgo, PtaMAgo, PtbMAgo, CsiMAgo, MtMAGO and GmMAGO, while the HbY14b protein shared 91\%, $80 \%, 71 \%, 75 \%, 67 \%$ and $69 \%$ amino acid identity with the homologs of RcMAGO, PtaMAGO, PtbMAGO, CsiMAGO, MtMAGO and GmMAGO. The Y14s protein possessed seven $\beta$-strands $(\beta 1-\beta 7)$ and two $\alpha$-helices $(\alpha 1$ $\alpha 2$ ) in a $\beta 1-\beta 2-\alpha 1-\beta 3-\beta 4-\beta 5-\alpha 2-\beta 6-\beta 7$ arrangement (Figure 1B). Alignment of the Y14 proteins revealed a wellconserved RNA-binding domain (RBD) in the middle of the protein (Figure 1B). An exon-intron structure analysis revealed that HbMAGOs consisted of three introns and two exons, while the HbY14s had three introns and four exons, respectively (Figure $1 \mathrm{C}$ ). Although, the differences in the cDNA sequences between two MAGOs or two Y14s were small, the differences in genomic DNA sequences were enormous.

\section{Phylogenetic analysis of the rubber tree HbMAGO and $\mathrm{HbY} 14$ families}

To obtain information about the evolutionary relationships of HbMAGOs and HbY14s, phylogenetic analyses were done based on multiple sequence alignments of all transcribed proteins from these two gene families in plants. Phylogenetic analysis based on the alignments of MAGO family proteins showed that rubber trees and other dicots were clustered in same group (Figure 2A), whereas members of the Y14 family clustered with other dicots in a single group (Figure 2B). These analyses also revealed that the MAGO and Y14 genes were present in algae and that their differentiation accompanied that of the corresponding species.

\section{Subcellular localization of HbMAGOs and HbY14s}

MAGO and Y14 are nucleocytoplasmic shuttling proteins located predominantly in the nucleoplasm and nuclear speckles (Micklem et al., 1997; Kataoka et al., 2000; Mohr et al., 2001). To confirm the subcellular location of HbMAGOs and $\mathrm{HbY} 14 \mathrm{~s}$, the fusion proteins and the GFP control constructs were introduced into onion epidermal cells by agroinfiltration using a water circulating vacuum pump and observed under a fluorescence mi- 
croscope. HbMAGOs and HbY14s were targeted the GFP control protein was distributed throughout the exclusively to the nucleus of the epidermal cells, whereas cells (Figure 3).
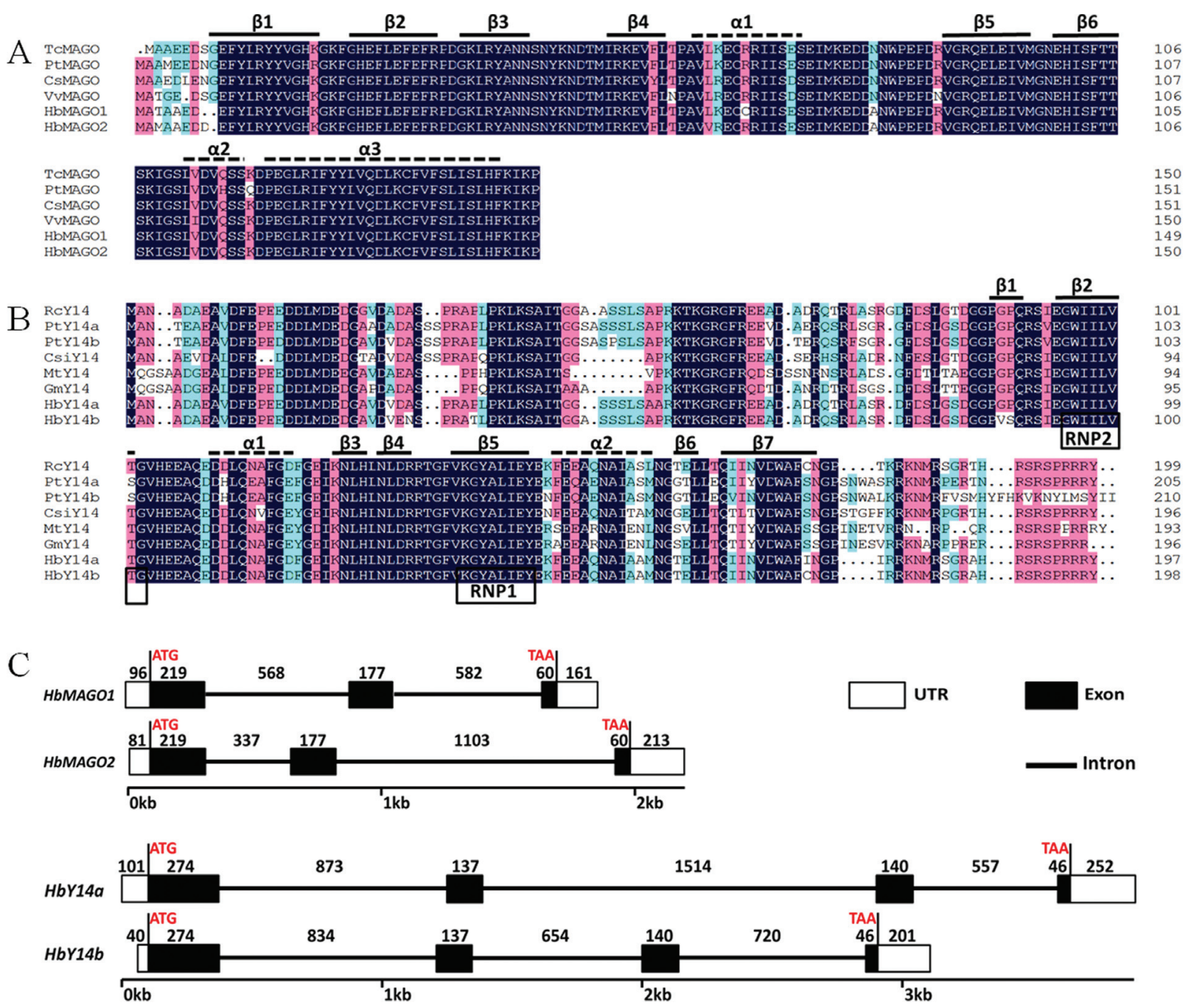

Figure 1 - Sequence alignment of the deduced HbMAGOs (A) and HbY14s (B), and intron-exon organization and exon length (C). Amino acid residues that are identical in the sequences are darkly shaded and well-conserved residues are shaded in pink. The $\alpha$-helices and $\beta$-strands of the HbMAGOs and $\mathrm{HbY} 14 \mathrm{~s}$ are shown as dashed lines and solid lines, respectively. The exons are shown as boxes (open reading frame in black, untranslated region (UTR) in white) and the introns are represented by lines.

Table 2 - Characterization of identified HbMAGO and HbY14 families.

\begin{tabular}{|c|c|c|c|c|c|c|c|c|c|c|c|c|}
\hline \multirow[b]{2}{*}{ Gene name } & \multirow{2}{*}{$\begin{array}{l}\text { GenBank } \\
\text { (AJJZ010000000) }\end{array}$} & \multirow{2}{*}{$\begin{array}{l}\text { Gene } \\
\text { (bp) }\end{array}$} & \multirow{2}{*}{$\begin{array}{l}\text { Exon } \\
\text { number }\end{array}$} & \multicolumn{5}{|c|}{ Exon length (bp) } & \multirow{2}{*}{$\begin{array}{l}\text { ORF } \\
\text { (bp) }\end{array}$} & \multicolumn{3}{|c|}{ Protein } \\
\hline & & & & E1 & E2 & E3 & E4 & 3'UTR & & aa & MW & pI \\
\hline$H b M A G O 1$ & $\begin{array}{l}\text { AJJZ010970869.1 } \\
\text { AJJZ011020394.1 }\end{array}$ & 1863 & 3 & 219 & 177 & 60 & & 161 & 456 & 151 & 17,476 & 5.68 \\
\hline$H b M A G O 2$ & $\begin{array}{l}\text { AJJZ010203456.1 } \\
\text { AJJZ010251509.1 }\end{array}$ & 2190 & 3 & 219 & 177 & 60 & & 213 & 456 & 151 & 17,688 & 5.69 \\
\hline$H b Y 14 a$ & $\begin{array}{l}\text { AJJZ010172128.1 } \\
\text { AJJZ010296629.1 }\end{array}$ & 3894 & 4 & 274 & 137 & 140 & 46 & 252 & 597 & 198 & 21,737 & 4.79 \\
\hline$H b Y 14 b$ & $\begin{array}{l}\text { AJJZ010209074.1 } \\
\text { AJJZ010209073.1 }\end{array}$ & 3046 & 4 & 274 & 137 & 140 & 46 & 201 & 597 & 198 & 21,903 & 4.79 \\
\hline
\end{tabular}

aa - amino acids, ORF - open reading frame. 


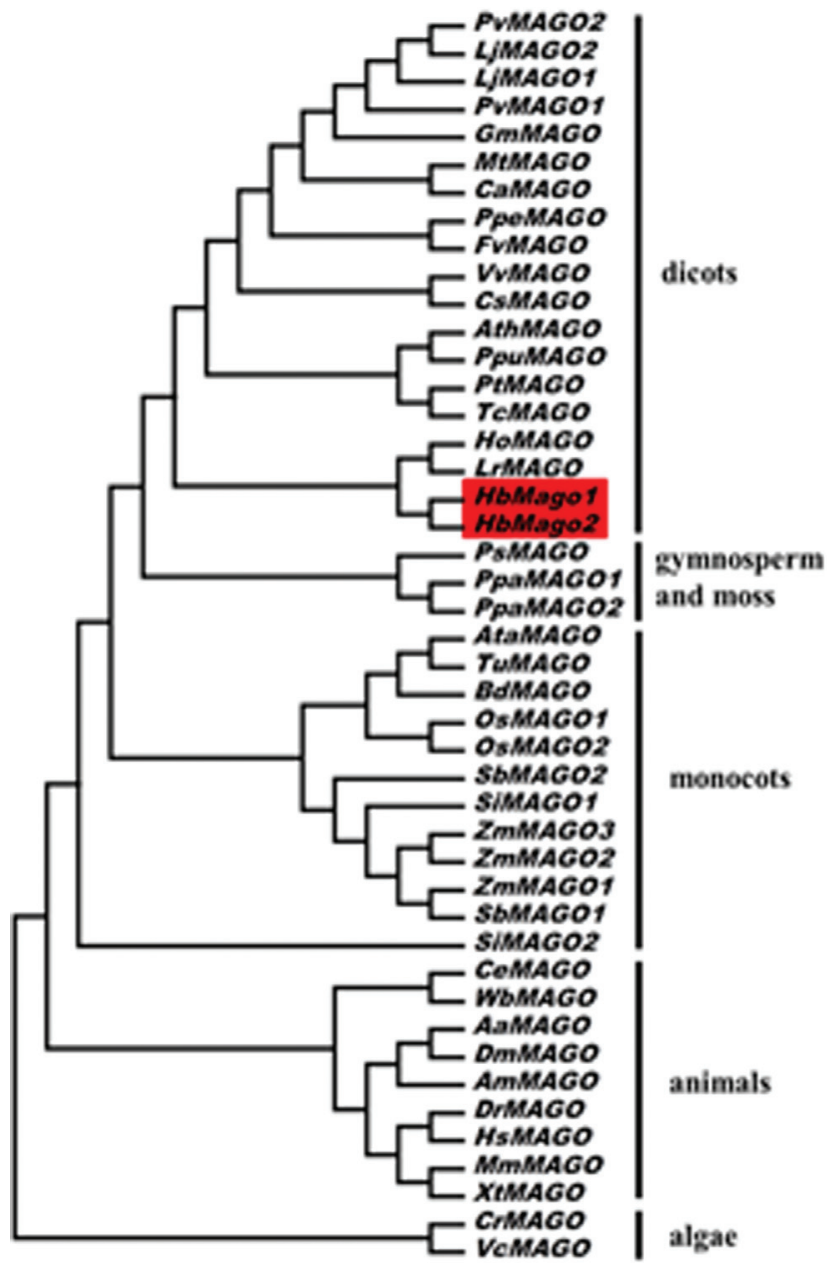

A

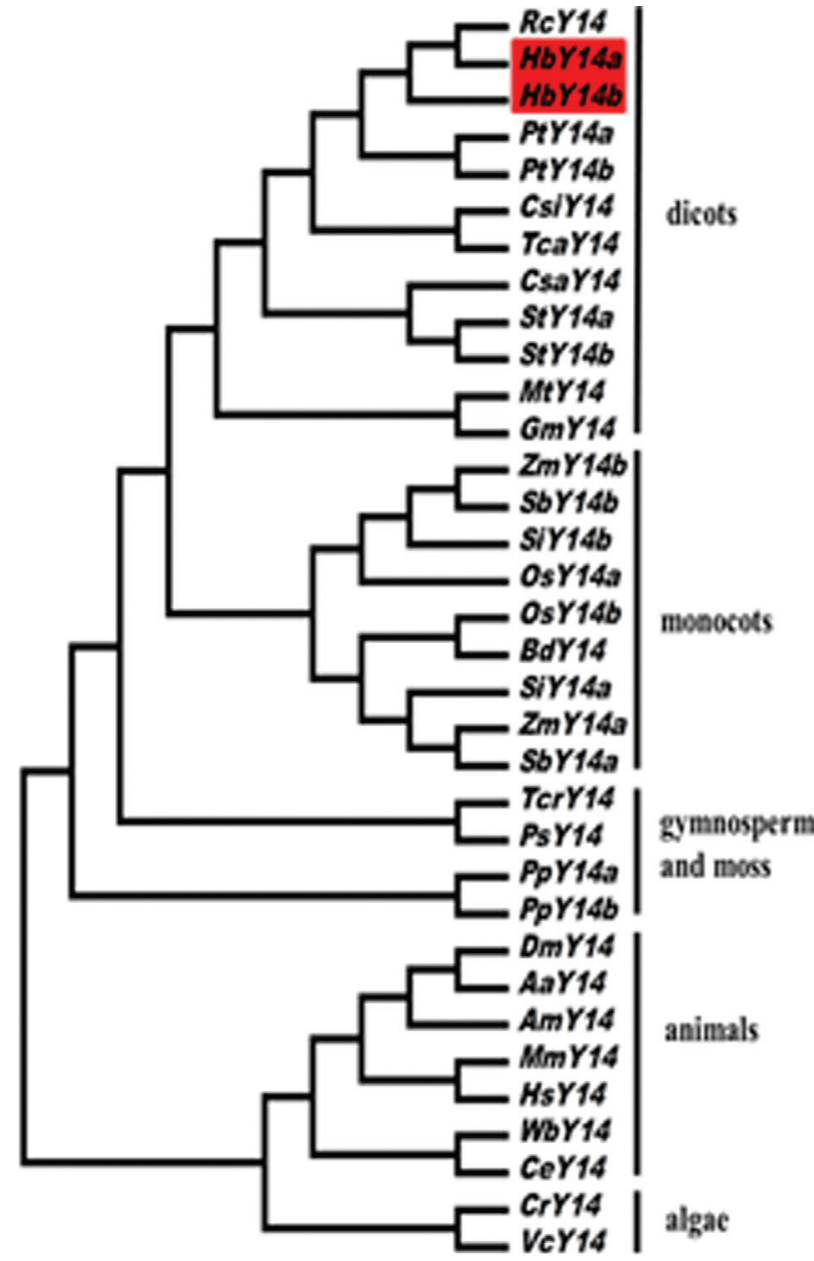

B

Figure 2 - Phylogenetic trees of HbMAGOs (A) and HbY14s (B). The trees were calculated based on the HbMAGO and HbY14 protein sequences and other plant MAGOs and Y14s. The GenBank accession numbers of selected homologs used to produce the phylogenetic trees are: CeMAGO (NP_493025.1), AaMAGO (XP_001660832.1), AmMAGO (XP_001120074.1), DmMAGO (NP_476636.1), WbMAGO (EJW84410.1), HsMAGO (NP_002361.1), MmMAGO (NP_079840.2), DrMAGO (NP_001017700.1), XtMAGO (XP_002931471.1), CrMAGO (XP_001694745.1), VcMAGO (XP_002954749.1), OsMAGO1 (EEC82788.1), OsMAGO2 (NP_001066589.1), ZmMAGÖ1 (NP_001145913.1), ZmMĀGO2 (NP_001146966.1), ZmMAGO3 (ACG28070.1), SiMAGO1 (XP 004972442.1), SiMAGO2 (XP 004957130.1), BdMAGO (XP 003573269.1), AtaMAGO (EMT32727.1), TuMAGO (EMS54552.1), SbMĀGO1 (XP_002443724.1), SbMAGO2 (XP_002462539.1), AthMAGO (NP_171716.1), TcMAGO (XP_007052335.1), PtMAGO (XP_006375160.1), LrMAGO (ACT33369.1), PpeMAGO (XP_007220423.1), CsMAGO (XP_004133764.1), PpuMAGO (ABQ11262.1), VvMAGO (XP_002281294.1), CaMAGO (XP_004501210.1), MtMAGÖ (ACJ86076.1), GmMAGO (NP_001236090.1), LjMAGO1 (AFK33465.1), LjMAGO2 (AFK48815.1), PvMAGO1 (XP_007147870.1), PvMAGO2 (XP_007134432.1), HoMAGO (AAS20975.1), FvMAGO (XP 004306981.1), PsMAGO (ABK22137.1), PpaMAGO1 (XP 001770408.1), PpatMAGO2 (XP 001763801.1), CeY14 (NP 497891.1), AaY14 (XP_001652167.1), AmY14 (XP_395245.2), DmY14 (NP_610454.2), WbY14 (EJW88540.1), HsY14 (NP_005096.1), MmY14 (NP 001095877.1), CrY14 (XP 002953417.1), CrY14 (XP 001696992.1), OsY14a (NP 001051661.1), OsY14b (XP 006654975.1), ZmY14a (NP_001150559.1), ZmY14b (NP_001150263.1), SiY14a (XP_004960437.1), SiY14b (XP_004981327.1), BdY14 (XP_003568971.1), SbY14a (XP 002439249.1), SbY14b (XP 002466233.1), RcY14 (XP 002513523.1), PtY14a (XP 002299789.1), PtY14b (XP 002314085.2), CsiY14 (XP_006470660.1), MtY14 (XP_003610955.1), GmY14 (XP_003517521.1), TcaY14 (XP_007015222.1), CsaY14 (XP_004139049.1), StY14a (XP 006353634.1), StY14b (NP 001274809.1), TcrY14 (ABB91897.1), PsY14 (ABK25331.1), PpY14a (XP_001758256.1) and PpY14b (XP_001771298.1)

Expression analysis of $H b M A G O s$ and $H b Y 14 s$ in different tissues

Real-time quantitative PCR was used to examine the expression patterns of HbMAGOs and HbY14s in bark, flowers, latex, leaves and roots. The expression profiles showed that the four genes (two each for HbMAGO and HbY14) were transcribed in all of the tissues examined, with the highest transcription in latex; for HbMAGO1, the level of transcription in bark was similar to that seen in latex (Figure 4A). 


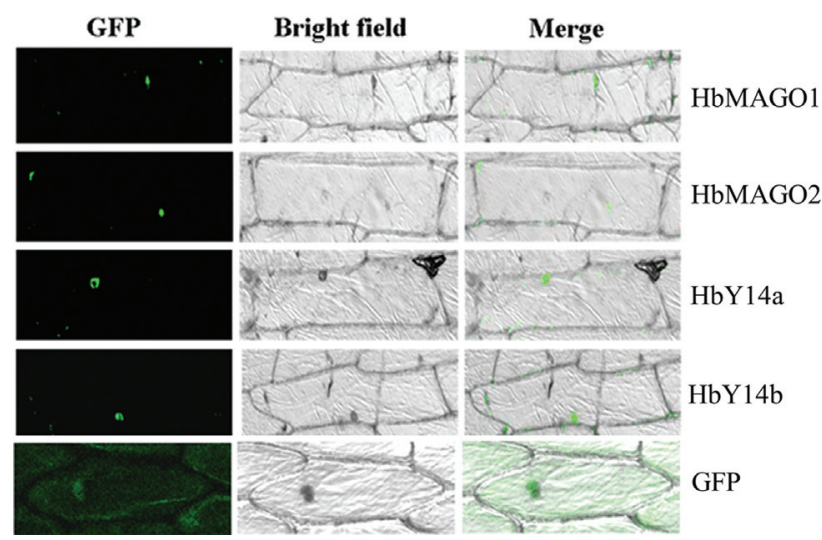

Figure 3 - Subcellular localization of HbMAGOs and HbY14s. Bottom row of panels: fluorescence, bright field and merged fluorescence images of the GFP control. The other columns indicate the corresponding fluorescence, bright field and merged fluorescence images of HbMAGOs and HbY14s.

\section{Expression patterns of $H b M A G O s$ and $H b Y 14 s$ in latex respond to treatment with $\mathrm{JA}$ and $\mathrm{ET}$}

Since ET and JA have an important role as signaling molecules that regulate rubber biosynthesis in rubber trees (Hao and $\mathrm{Wu}, 2000$; Zeng et al., 2009), we examined whether exposure to ET or JA could influence HbMAGO and $\mathrm{HbY} 14$ expression in latex (Figure 4B), despite the lack of evidence relating MAGOs and Y14s to hormone signaling pathways. HbMAGO1 was regulated by ET but not by $\mathrm{JA}$, and HbMAGO2 was induced by JA but not by ET. Two HbY14s were induced by ET and JA in latex. Gene expression increased significantly within $9 \mathrm{~h}$ and subsequently decreased when treated with the plant hormones, i.e., HbMAGO1 was influenced by ET, HbMAGO2 by JA, HbY14a by ET and JA, and HbY14b by JA; HbY14b expression also increased significantly within $24 \mathrm{~h}$ and subsequently decreased in response to ET.

\section{Interaction of HbMAGOs with $\mathrm{HbY} 14 \mathrm{~s}$}

As the core of EJC, MAGO and Y14 can form heterodimers. Figure 5A shows that HbMAGOs interacted with HbY14s in the YTH system. The interaction of HbMAGO1 with $\mathrm{HbY} 14 \mathrm{~s}$ was significantly greater than that of HbMAGO2 with HbY14s, as assessed by quantifying the $\beta$-galactosidase activity (Figure 5B). This finding suggests that HbMAGOl interacted specifically with HbY14s.

\section{Interaction of HbMAGO2 with $\mathrm{gp} 91^{\text {phox }}$}

Using BK-MAGO as bait, we obtained gp91 $1^{\text {phox }}$ as one of the prey proteins screened from a two-hybrid latex library (Figure 6A). The open reading frame of gp91 $1^{\text {phox }}$ was cloned (GeneBank: AJJZ010935968.1). To confirm the subcellular location of gp9 $1^{\text {phox }}$ proteins, gp91 ${ }^{\text {phox }}$ :GFP fusion proteins were transiently expressed in onion epidermal cells. Confocal imaging of GFP fluorescence revealed
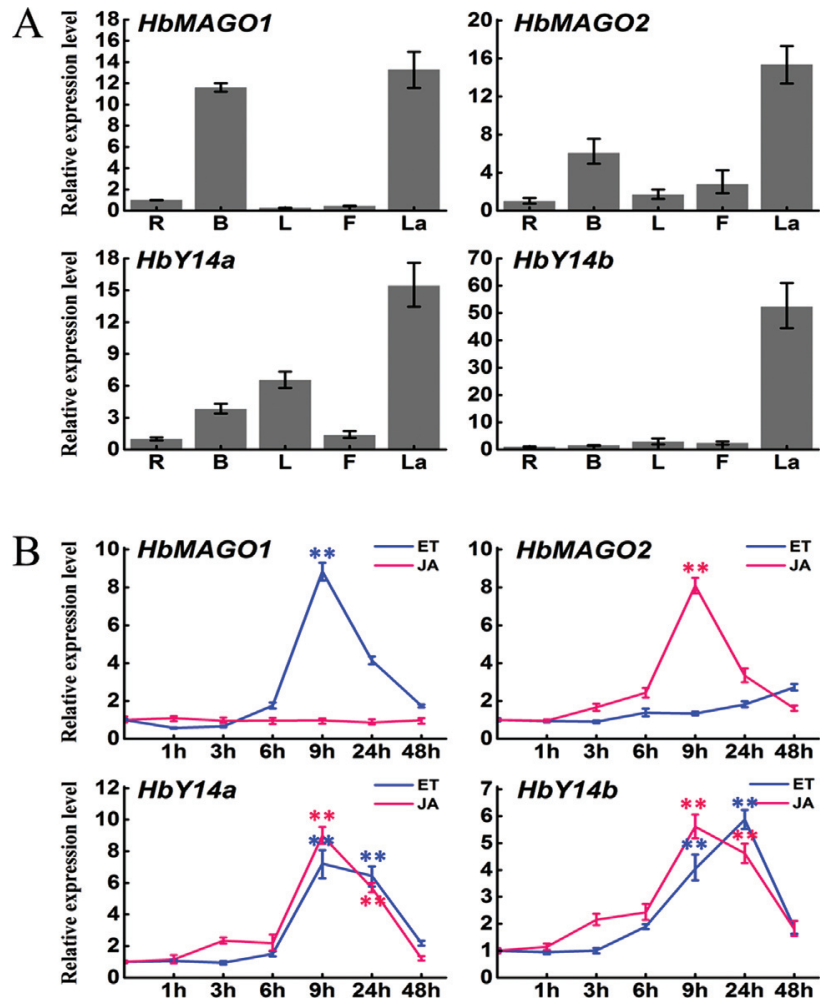

Figure 4 - Expression patterns of $H b M A G O s$ and $H b Y 14 s$ in different tissues (A) and the responses to treatment with jasmonic acid (JA) and ethylene (ET) (B). Relative transcript abundances of HbMAGOs and $\mathrm{HbY} 14 \mathrm{~s}$ were examined by RT-qPCR. The Y-axis indicates the relative transcript abundance level, while the $\mathrm{X}$-axis denotes the rubber tree tissues examined (A) and the time course of the response to treatment with ET and JA (B). The rubber tree actin gene (GenBank HQ260674.1) was used as an internal control. PCR primers were designed to avoid the conserved region and to amplify 100-300 bp products. The primer sequences are shown in Table 2. B - bark, L - leaves, $\mathrm{F}$ - flowers, $\mathrm{La}$ - latex and $\mathrm{R}$ - roots. **p $<$ 0.01 (ANOVA).

that the gp91 ${ }^{\text {phox }}$ GFP fusion protein was located in the cytomembrane (Figure 6B). The possible interaction between $\mathrm{HbMAGO} 2$ and $\mathrm{gp} 91^{\text {phox }}$ was examined using a $\mathrm{BiFC}$ assay. A strong fluorescence signal was observed in the cytomembrane of epidermal cells under normal conditions (Figure 6C) and during plasmolysis (Figure 6D), but not with the negative controls. These data confirmed the interaction between $\mathrm{HbMAGO} 2$ and gp9 $1^{\text {phox }}$.

\section{Discussion}

There is increasing evidence that many factors involved in basic cellular processes in animals and plants are conserved. MAGO and Y14 proteins, which are important for cellular differentiation in animals, show marked sequence conservation across a wide range of species (Chen et al., 2007). The MAGO and Y14 proteins are encoded by a small gene family in plants. The Arabidopsis genome contains one MAGO and one Y14 gene (Park and Muench, 2007), whereas the rice genome contains two MAGO and two Y14 genes (Gong and He, 2014). We have identified 


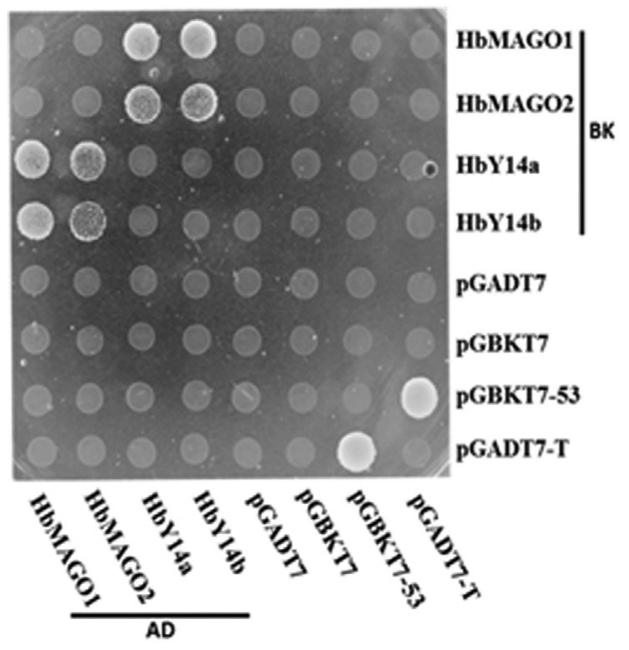

A

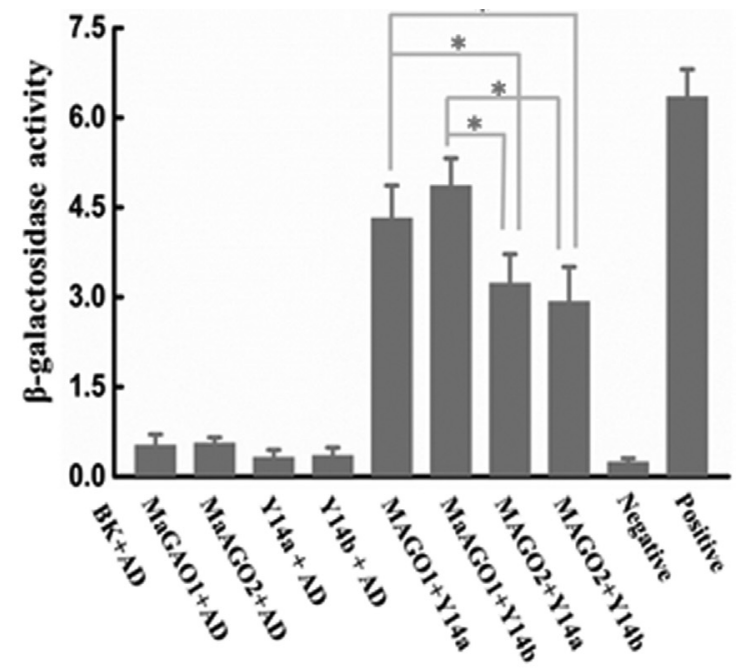

B

Figure 5 - Protein interaction matrices for HbMAGO and HbY14 proteins (A) and quantification of $\beta$-galactosidase activity (B). The combination of bait proteins (BD) and prey proteins (AD) is indicated. Interactions between HbMAGOs and HbY14s were detected in yeast and the same amounts of co-transformed yeast cells were grown in the highest stringent conditions (QDO/X/A medium). Transformants containing BK-53 and AD-T were used as positive controls and those containing only $\mathrm{BK}$ and $\mathrm{AD}$ or fused proteins with $\mathrm{BK}$ or $\mathrm{AD}$ only were used as negative controls (A). In panel (B), $\beta$-galactosidase activity was used as an indicator of the interaction between HbMAGOs and HbY 14s (B). Representative results obtained in at least three independent experiments are shown. The columns in panel (B) represent the mean \pm SEM. *p $<0.05$ (ANOVA).

two MAGO and two Y14 genes in the rubber tree genome. MAGO and Y14 proteins are highly conserved among eukaryotes, suggesting that the corresponding genes are essential for eukaryotes. Y14 proteins have a well-conserved $\mathrm{RBD}$ in the middle region. The RBD is a characteristic feature of the ribonucleo-protein (RNP) family of RNA binding proteins and is evolutionarily conserved (Nagai et al., 1995). The homologs of Y14 that have these characteristics are known to be involved in RNA localization, mRNA splicing and exon-exon junctions (Kim et al., 2001; Mohr et al., 2001; Chuang et al., 2013). It has been suggested that HbY14 may also have these critical functions in plants. HbMAGOs and HbY14s shared high amino acid sequence identity with homologs from other species. Although MAGO and Y14 are highly conserved in eukaryotes, the clusters of MAGO and Y14 are easily distinguished between dicots and other clades. The evidence from gene structure also supports this conclusion. The two MAGO genes in humans (MAGOH) consist of five exons with a conserved length and structure, but the coding sequence of MAGOHB is longer than that of MAGOH (Singh et al., 2013).

MAGO and Y14 are nucleocytoplasmic shuttling proteins located predominantly in the nucleoplasm and nuclear speckles (Micklem et al., 1997; Kataoka et al., 2000; Mohr et al., 2001). In Taiwania cryptomerioides, TcMAGO-EGFP and TcY14-EGFP were detected at low levels in the cytoplasm at $20 \mathrm{~h}$ and $22 \mathrm{~h}$. After $22 \mathrm{~h}$, TcMAGO-EGFP was located only in the nuclei, whereas TcY14-EGFP was present in the nuclei and at low levels in the cytoplasm (Chen et al., 2007). In rice, OsMAGO2 and
OsY14b showed identical distributions in cells and were located in the nuclei and cytoplasm. Surprisingly, OsY14a seemed to be uniquely located in the nuclei, in contrast to the established subcellular localization of these EJC subunits (Gong and He, 2014). As shown here, HbMAGOs and $\mathrm{HbY} 14 \mathrm{~s}$ were located in the nuclei, in a manner similar to their homologs in other species.

In rubber trees, laticifers are tissues that are specifically involved in the biosynthesis and storage of natural rubber, as well as in defense against pathogens. In this study, we found that HbMAGOs and HbY14s had a similar, constitutive expression pattern in different tissues of rubber tree. Four genes were transcribed in the tissues examined, with the highest transcription occurring in latex, a finding indicative of the vital role of these genes in laticifer cells. HbMAGO1 was regulated by ET but not by JA, and HbMAGO2 was induced by JA but not by ET. In contrast, two $\mathrm{HbY} 14 \mathrm{~s}$ were regulated by ET and JA in latex. The biosynthesis of natural rubber is enhanced in rubber trees by the endogenous accumulation and exogenous application of JA (Hao and $\mathrm{Wu}, 2000$ ). Our findings suggest that the HbMAGO and HbY14 genes examined may regulate natural rubber biosynthesis in rubber tree laticifer cells via ET and JA signal transduction pathways. The differences in the levels of transcription of the genes in response to ET and JA also indicated functional differences in laticifer cells. This is the first demonstration that HbMAGO and HbY14 are linked to hormone signaling pathways. The precise relationship will be investigated in future studies.

The MAGO gene was first identified as a strict maternal effect gene in Drosophila, where a single point mutant 


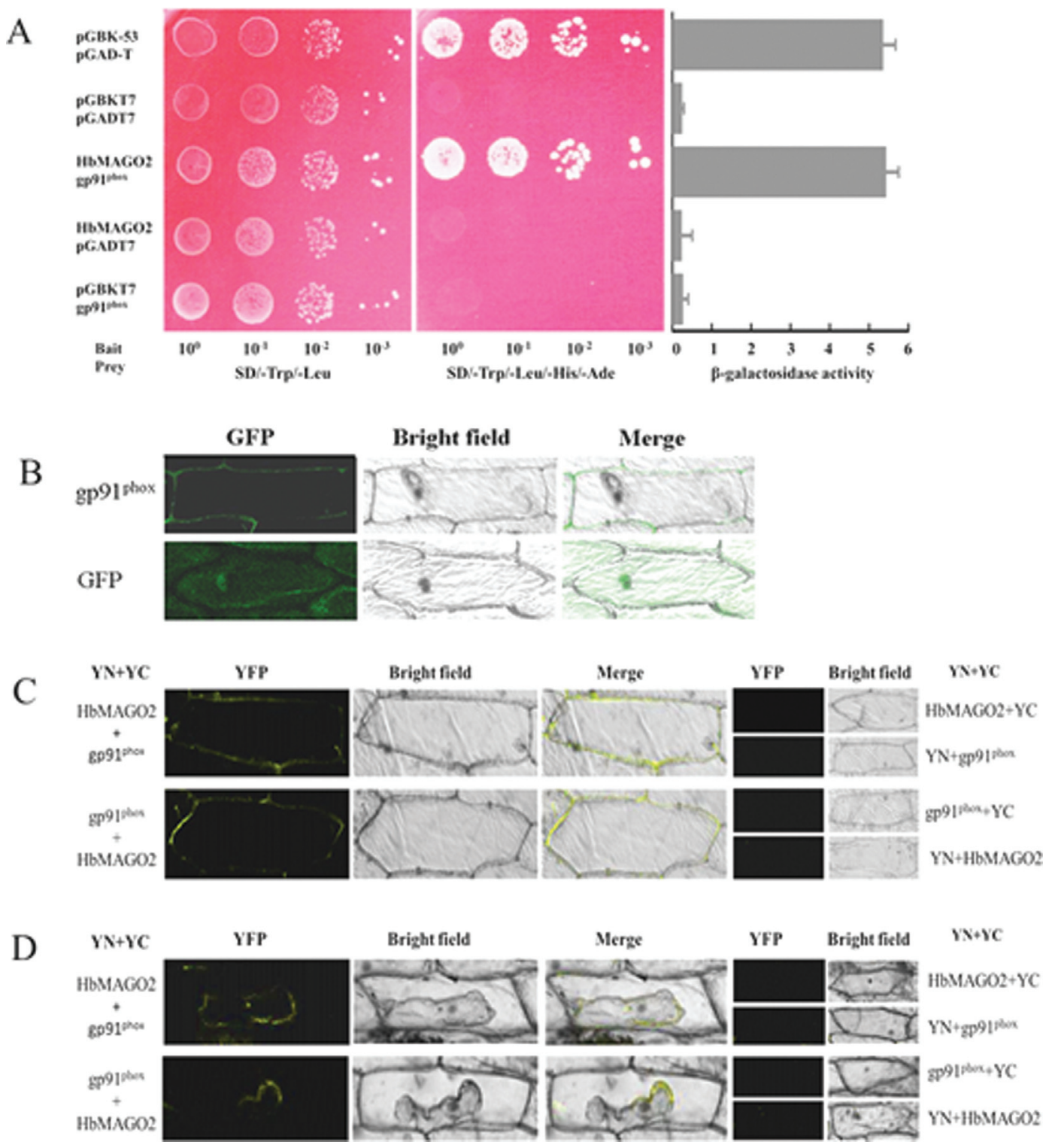

Figure 6 - Functional analysis of HbMAGO2. Interaction of HbMAGO2 with gp9 $91^{\text {phox }}$ in yeast (A), subcellular localization of gp91 ${ }^{\text {phox }}(\mathrm{B})$, and bimolecular fluorescence complementation (BiFC) assays in plants (C,D). pGBK-HbMAGO2 (bait) and pGAD-gp91 ${ }^{\text {phox }}$ (prey) were co-transformed into AH109 yeast cells. Aliquots $(10 \mu \mathrm{L})$ of a 10 diluted yeast suspension culture co-transformed with bait and prey constructs was spotted onto SD/-Trp/-Leu and $\mathrm{SD} /$-Trp/-Leu/-His/-Ade selection plates. Negative controls consisted of vector with only BK and AD or fused proteins with BK or AD. The intensity of the interaction was assessed by assaying $\beta$-galactosidase activity (A). Panel (B) shows the subcellular localization of gp9 $1^{\text {phox }}$. The interaction between $\mathrm{HbMAGO} 2$ and gp9 ${ }^{\text {phox }}$ was confirmed using the BiFC assay (C). Epidermal cells were co-transformed with HbMAGO2 and gp91 $1^{\text {phox }}$ proteins fused to the $\mathrm{N}$ - or C-terminal half of yellow fluorescent protein. $\mathrm{HbMAGO} 2$ or $\mathrm{gp} 91^{\text {phox }}$ with vector were used as negative controls. Onion epidermal cells were observed under normal conditions (C) and after plasmolysis (incubation for $30 \mathrm{~min}$ in a $30 \%$ sucrose solution) (D). Representative results obtained in at least three independent experiments are shown. The columns in panel (A) represent the mean \pm SEM.

in the MAGO locus gave rise to a grandchildless phenotype because of a defect in the correct cytoplasmic location of oskar mRNA (Boswell et al., 1991; Newmark and Boswell, 1994; Micklem et al., 1997; Newmark et al., 1997). MAGO always functions together with an RNA-binding protein, known as Y14 in Xenopus (Kataoka et al., 2000), RBM8A in humans (Zhao et al., 2000) and Tsunagi in Drosophila (Mohr et al., 2001); this protein shuttles between the nu- cleus and cytoplasm (Hachet and Ephrussi, 2001; Kim et al., 2001).

The MAGO-Y14 complex is the core of the EJC assembled on RNA 20 nucleotides upstream of exon-exon junctions (Kataoka et al., 2001; Bono et al., 2004). Determination of the crystal structure of the MAGO-Y14 complex showed that the MAGO-Y14 interaction was highly specific and strongly conserved (Zhao et al., 2000; Kataoka et al., 2001; Le et al., 2001; Shi and Xu, 2003; Stroupe et 
al., 2006). In plants, the MAGO-Y14 complex has also been found in T. cryptomerioides and rice (Chen et al., 2007; Gong and He, 2014). The functional barrier between the two protein families was not observed within dicots or duplicates of rice, but was observed between dicots and monocot or plants and animals (Gong et al., 2014). The high specificity and conservation of the MAGO-Y14 interaction is clade-specific, and such co-evolution allows the interaction between these proteins to be maintained across large evolutionary time scales. In this work, we identified HbMAGO1/2 and HbY14a/b homologs derived from rubber tree latex and confirmed the interaction between HbMAGO and HbY14 using YTH analysis. Overall, our findings indicate that $\mathrm{HbMAGO} / 2$ and $\mathrm{HbY} 14 \mathrm{a} / \mathrm{b}$ are evolutionarily highly conserved proteins with similar functions to their homologs.

In rubber trees, NADPH (nicotinamide adenine dinucleotide phosphate) oxidase is involved in the accumulation of reactive oxygen species (ROS) in tapping panel dryness (TPD), a physiological disorder characterized by the spontaneous drying up of the tapping cut that results in an abnormally low yield or stoppage of latex flow (Jacob et al., 1994; Chen et al., 2003; Venkatachalam et al., 2009). ROS are involved in the coagulation of rubber particles that dramatically reduces natural rubber production. High ROS production in latex cells triggers oxidative stress leading to the in situ coagulation of rubber particles (Chrestin et al., 1984; Li et al., 2010; Leclercq et al., 2012). ROS are also important signaling molecules in plants.

ROS formation is one of the early physiological responses of plant cells to biotic and abiotic stress, such as wounding and pathogens (Bogre et al., 1997; Bolwell et al., 2002). NADPH oxidase is the main source of ROS in plants (Asai et al., 2008). In animals, the NADPH oxidase complex consists of two plasma membrane proteins, gp91 ${ }^{\text {phox }}$ (phox for phagocyte oxidase) and $\mathrm{p} 22^{\text {phox }}$. Cytosolic regulatory proteins $\mathrm{p} 47^{\text {phox }}, \mathrm{p} 67^{\text {phox }}, \mathrm{p} 40^{\text {phox }}$ and Rac2, translocate to the plasma membrane to form the active complex after stimulation (Brandes et al., 2014). However, no homologs of the $\mathrm{p} 22^{\text {phox }}, \mathrm{p} 67^{\text {phox }}, \mathrm{p} 47^{\text {phox }}$ and $\mathrm{p} 40^{\text {phox }}$ regulators of phagocyte NADPH oxidase were found in plants.

Plant NADPH oxidases are known as respiratory burst oxidase homologs (RBOHs) and are homologous to the catalytic subunit (gp91 ${ }^{\text {phox }}$ ) of mammalian NADPH oxidases (Rodriguez et al., 2007; Suzuki et al., 2011). RBOHs have been identified and characterized in several species, including Arabidopsis thaliana (Kawarazaki et al., 2013), potato (Chen et al., 2013), rice (Yoshie et al., 2005), wheat (Yamauchi et al., 2014). In TPD trees, high levels of NADPH oxidase activities lead to the release of $\mathrm{O}_{2}{ }^{-}$, a toxic form of oxygen (Jacob et al., 1994). Cellular membranes and especially lutoids, which contain coagulant factors involved in the aggregation of rubber particles, are damaged by $\mathrm{O}_{2}^{-}$(Chrestin et al., 1984; Li et al., 2010; Leclercq et al., 2012).
In plants, MAGO can participate in biological processes through protein-protein interactions. In Physalis, MAGO interacts with a MADS-Domain protein that can regulate plant development through the formation of dimers and higher order complexes to influence male fertility and calyx development (He et al., 2007); this finding suggests that MAGO plays a role in plant biological processes independently of the EJC. Little is known about the function of HbMAGO mediated by interaction with target proteins in rubber trees. As shown here, the $\mathrm{YTH}$ and $\mathrm{BiFC}$ assays indicated that $\mathrm{HbMAGO} 2$ interacted with $\mathrm{gp} 91^{\text {phox }}$. This finding suggests that $\mathrm{HbMAGO} 2$ may regulate NADPH oxidase-dependent oxidative bursts in rubber tree latex via protein-protein interactions.

Functional studies have shown that MAGO is involved in development of organization in multicellular organisms. Over-expression or knockdown of MAGO results in phenotypic alterations that vary among plants, including visible longer roots and a more complex root system, a greater number of leaves, short and fasciated stems, short lateral roots and non-viable seeds, and short rice plants with abnormal flowers (Chen et al., 2007; Park et al., 2009; Gong and He, 2014). In Drosophila, the EJC controls the splicing of mapk and other long intron-containing transcripts and mitogen-activated protein kinase (MAPK) signaling depends on the regulation of MAPK levels by the EJC (Ashton-Beaucage et al., 2010). During development of the eye, MAPK is the primary functional target of MAGO (Roignant and Treisman, 2010). As core components of the EJC linked to MAPK signaling pathway, MAGOs can control animal cell or tissue development. Laticifer cells are some of the most important plant cells that continuously produce natural rubber and are arranged as concentric sheaths in the phloem (Chen et al., 2000; Han et al., 2000; Pickard, 2008). There are few reports on the regulation of laticifer development in general (Tan et al., 2014), and the development of laticifer cells in rubber trees remains poorly understood. Our findings suggest that MAGO may regulate the development of laticifer cells, although further experiments are need to confirm this suggestion.

\section{Conclusions}

The present study has provided a comprehensive genomic analysis of the rubber tree HbMAGO and HbY14 gene families and the first evidence that HbMAGO and HbY14 are linked with hormone signaling pathways, including rubber particle aggregation in laticifer cells. These data provide important insights into the potential roles of HbMAGO and HbY14 gene families in rubber trees and may contribute to clarifying the function of $\mathrm{HbMAGO} 2$ in the aggregation of natural rubber particles. 


\section{Acknowledgments}

This work was supported by the National Natural Science Foundation of China (grant no. 31170634) and Innovation Subject of Hainan province (grants nos. B201301 and Hyb2014-03).

\section{References}

Asai S, Ohta K and Yoshioka H (2008) MAPK signaling regulates nitric oxide and NADPH oxidase-dependent oxidative bursts in Nicotiana benthamiana. Plant Cell 20:1390-1406.

Ashton-Beaucage D, Udell CM, Lavoie H, Baril C, Lefrancois M, Chagnon P, Gendron P, Caron-Lizotte O, Bonneil E, Thibault $\mathrm{P}$, et al. (2010) The exon junction complex controls the splicing of mapk and other long intron-containing transcripts in Drosophila. Cell 143:251-262.

Bolwell GP, Bindschedler LV, Blee KA, Butt VS, Davies DR, Gardner SL, Gerrish C and Minibayeva F (2002) The apoplastic oxidative burst in response to biotic stress in plants: a three-component system. J Exp Bot 53:1367-1376.

Bogre L, Ligterink W, Meskiene I, Barker PJ, Heberle-Bors E, Huskisson NS and Hirt H (1997) Wounding induces the rapid and transient activation of a specific MAP kinase pathway. Plant Cell 9:75-83.

Bono F, Ebert J, Unterholzner L, Güttler T, Izaurralde E and Conti E (2004) Molecular insights into the interaction of PYM with the Mago-Y14 core of the exon junction complex. EMBO Rep 5:304-310.

Boothby TC and Wolniak SM (2011) Masked mRNA is stored with aggregated nuclear speckles and its asymmetric redistribution requires a homolog of Mago nashi. BMC Cell Biol 12:45.

Boswell RE, Prout ME and Steichen JC (1991) Mutations in a newly identified Drosophila melanogaster gene, mago nashi, disrupt germ cell formation and result in the formation of mirror-image symmetrical double abdomen embryos. Development 113:373-384.

Brandes RP, Weissmann N and Schroder K (2014) Nox family NADPH oxidases: molecular mechanisms of activation. Free Radical Biol Med 76c:208-226.

Chen DH, Ye HC, Li GF and Liu Y (2000) Advances in molecular biology of plant isoprenoid metabolic pathway. Acta Bot Sin 42:551-558.

Chen HJ, Huang CS, Huang GJ, Chow TJ and Lin YH (2013) NADPH oxidase inhibitor diphenyleneiodonium and reduced glutathione mitigate ethephon-mediated leaf senescence, $\mathrm{H}_{2} \mathrm{O}_{2}$ elevation and senescence-associated gene expression in sweet potato (Ipomoea batatas). J Plant Physiol 170:1471-1483.

Chen SC, Peng SQ, Huang GX, Wu KX, Fu XH and Chen ZQ (2003) Association of decreased expression of a Myb transcription factor with the TPD (tapping panel dryness) syndrome in Hevea brasiliensis. Plant Mol Biol 51:51-58.

Chen YR, Shaw JF, Chung MC and Chu FH (2007) Molecular identification and characterization of Tcmago and TcY14 in Taiwania (Taiwania cryptomerioides). Tree Physiol 27:1261-1271.

Chrestin H, Bangratz J, d'Auzac J and Jacob JL (1984) Role of the lutoidic tonoplast in the senescence and degeneration of the laticifers of Hevea brasiliensis. Zeitschrift für Pflanzenphysiol 114:261-268.

Chuang TW, Chang WL, Lee KM and Tarn WY (2013) The RNA-binding protein Y14 inhibits mRNA decapping and modulates processing body formation. Mol Biol Cell 24:1-13.

Fribourg S, Gatfield D, Izaurralde E and Conti E (2003) A novel mode of RBD-protein recognition in the Y14-Mago complex. Nat Struct Biol 10:433-439.

Gehring NH, Lamprinaki S, Kulozik AE and Hentze MW (2009) Disassembly of exon junction complexes by PYM. Cell 137:536-548.

Gong PC, Zhao M and He CY (2014) Slow co-evolution of the MAGO and Y14 protein families is required for the maintenance of their obligate heterodimerization mode. PLoS One 9:e84842.

Gong PC and He CY (2014) Uncovering divergence of rice exon junction complex core heterodimer gene duplication reveals their essential role in growth, development, and reproduction. Plant Physiol 165:1047-1061.

Hachet O and Ephrussi A (2001) Drosophila Y14 shuttles to the posterior of the oocyte and is required for oskar mRNA transport. Curr Biol 11:1666-1674.

Hao BZ and Wu JL (2000) Laticifer differentiation in Hevea brasiliensis: induction by exogenous jasmonic acid and linolenic acid. Ann Bot 85:37-43.

Han KH, Shin DH, Yang J, Kim IJ, Oh SK and Chow KS (2000) Genes expressed in the latex of Hevea brasiliensis. Tree Physiol 20:503-510.

He CY, Sommer H, Grosardt B, Huijser P and Saedler H (2007) PFMAGO, a MAGO NASHI-like factor, interacts with the MADS-domain protein MPF2 from Physalis floridana. Mol Biol Evol 24:1229-1241.

Jacob JL, Prevot JC and Lacrotte R (1994) Tapping panel dryness in Hevea brasiliensis. Plant Rech Dev 1:15-21.

Johnson MA, von Besser K, Zhou Q, Smith E, Aux G, Patton D, Levin JZ and Preuss D (2004) Arabidopsis hapless mutations define essential gametophytic functions. Genetics 168:971-982.

Kataoka N, Diem MD, Kim VN, Yong J and Dreyfuss G (2001) Magoh, a human homolog of Drosophila mago nashi protein, is a component of the splicing-dependent exon-exon junction complex. EMBO J 20:6424-6433.

Kawarazaki T, Kimura S, Iizuka A, Hanamata S, Nibori H, Michikawa M, Imai A, Abe M, Kaya H and Kuchitsu K (2013) A low temperature-inducible protein AtSRC2 enhances the ROS-producing activity of NADPH oxidase AtRbohF. Biochim Biophys Acta Mol Cell Res 1833:2775-2780.

Kataoka N, Yong J, Kim VN, Velazquez F, Perkinson RA, Wang F and Dreyfuss G (2000) Pre-mRNA splicing imprints mRNA in the nucleus with a novel RNA-binding protein that persists in the cytoplasm. Mol Cell 6:673-682.

Kim VN, Yong J, Kataoka N, Abel L, Diem MD and Dreyfuss G (2001) The Y14 protein communicates to the cytoplasm the position of exon-exon junctions. EMBO J 20:2062-2068.

Lau CK, Diem MD, Dreyfuss G and Van Duyne GD (2003) Structure of the Y14-Magoh core of the exon junction complex. Curr Biol 13:933-941.

Le HH and Seraphin B (2008) EJCs at the heart of translational control. Cell 133:213-216. 
Le HH, Nott A and Moore MJ (2003) How introns influence and enhance eukaryotic gene expression. Trends Biochem Sci 28:215-220.

Le HH, Izaurralde E, Maquat LE and Moore MJ (2000) The spliceosome deposits multiple proteins 20-24 nucleotides upstream of mRNA exon-exon junctions. EMBO J 19:6860-6869.

Le HH, Gatfield D, Braun IC, Forler D and Izaurralde E (2001) The protein Mago provides a link between splicing and mRNA localization. EMBO Rep 2:1119-1124.

Lee HC, Choe J, Chi SG and Kim YK (2009) Exon junction complex enhances translation of spliced mRNAs at multiple steps. Biochem Biophys Res Commun 384:334-340.

Leclercq J, Martin F, Sanier C, Clement-Vidal A, Fabre D, Oliver G, Lardet L, Ayar A, Peyramard M and Montoro P (2012) Over-expression of a cytosolic isoform of the $\mathrm{HbCuZnSOD}$ gene in Hevea brasiliensis changes its response to a water deficit. Plant Mol Biol 80:255-272.

Lejeune F and Maquat LE (2005) Mechanistic links between nonsense-mediated mRNA decay and pre-mRNA splicing in mammalian cells. Curr Opin Cell Biol 17:309-315.

Li DJ, Deng Z, Chen CL, Xia ZH, Wu M, He P and Chen SC (2010) Identification and characterization of genes associated with tapping panel dryness from Hevea brasiliensis latex using suppression subtractive hybridization. BMC Plant Biol 10:140.

Micklem DR, Dasgupta R, Elliott H, Gergely F, Davidson C, Brand A, Gonzalez-Reyes A and St Johnston D (1997) The mago nashi gene is required for the polarisation of the oocyte and the formation of perpendicular axes in Drosophila. Curr Biol 7:468-478.

Mohr SE, Dillon ST and Boswell RE (2001) The RNA-binding protein Tsunagi interacts with Mago Nashi to establish polarity and localize oskar mRNA during Drosophila oogenesis. Gene Dev 15:2886-2899.

Mufarrege EF, Gonzalez DH and Curi GC (2011) Functional interconnections of Arabidopsis exon junction complex proteins and genes at multiple steps of gene expression. J Exp Bot 62:5025-5036.

Nagai K, Oubridge C, Ito N, Avis J and Evans P (1995) The RNP domain: a sequence-specific RNA-binding domain involved in processing and transport of RNA. Trends Biochem Sci 20:235-240.

Newmark PA and Boswell RE (1994) The mago nashi locus encodes an essential product required for germ plasm assembly in Drosophila. Development 120:1303-1313.

Newmark PA, Mohr SE, Gong L and Boswell RE (1997) mago nashi mediates the posterior follicle cell-to-oocyte signal to organize axis formation in Drosophila. Development 124:3197-3207.

Park NI and Muench DG (2007) Biochemical and cellular characterization of the plant ortholog of PYM, a protein that interacts with the exon junction complex core proteins Mago and Y14. Planta 225:625-639.

Park NI, Yeung EC and Muench DG (2009) Mago Nashi is involved in meristem organization, pollen formation, and seed development in Arabidopsis. Plant Sci 176:461-469.

Pickard WF (2008) Laticifers and secretory ducts: two other tube systems in plants. New Phytol 177:877-888.

Rodriguez AA, Ramiro Lascano H, Bustos D and Taleisnik E (2007) Salinity-induced decrease in NADPH oxidase activ- ity in the maize leaf blade elongation zone. J Plant Physiol 164:223-230.

Roignant JY and Treisman JE (2010) Exon junction complex subunits are required to splice Drosophila MAP kinase, a large heterochromatic gene. Cell 143:238-250.

Shi H and Xu RM (2003) Crystal structure of the Drosophila Mago nashi-Y14 complex. Genes Dev 17:971-976.

Singh KK, Wachsmuth L, Kulozik AE and Gehring NH (2013) Two mammalian MAGOH genes contribute to exon junction complex composition and nonsense-mediated decay. RNA Biol 10:1291-1298.

Stroupe ME, Tange TO, Thomas DR, Moore MJ and Grigorieff N (2006) The three-dimensional architecture of the EJC core. J Mol Biol 360:743-749.

Suzuki N, Miller G, Morales J, Shulaev V, Torres MA and Mittler R (2011) Respiratory burst oxidases: the engines of ROS signaling. Curr Opin Plant Biol 14:691-699.

Tan DG, Sun XP and Zhang JM (2014) Age-dependent and jasmonic acid-induced laticifer-cell differentiation in anther callus cultures of rubber tree. Planta 240:337-344.

Tang CR, Qi JY, Li H, Zhang CL and Wang YK (2007) A convenient and efficient protocol for isolating high-quality RNA from latex of Hevea brasiliensis (para rubber tree). J Biochem Biophys Meth 70:749-754.

Tange TO, Nott A and Moore MJ (2004) The ever-increasing complexities of the exon junction complex. Curr Opin Cell Biol 16:279-284.

Tange TO, Shibuya T, Jurica MS and Moore MJ (2005) Biochemical analysis of the EJC reveals two new factors and a stable tetrameric protein core. RNA 11:1869-1883.

van der Weele CM, Tsai CW and Wolniak SM (2007) Mago nashi is essential for spermatogenesis in Marsilea. Mol Biol Cell 18:3711-3722.

Venkatachalam P, Thulaseedharan A and Raghothama K (2009) Molecular identification and characterization of a gene associated with the onset of tapping panel dryness (TPD) syndrome in rubber tree (Hevea brasiliensis Muell.) by mRNA differential display. Mol Biotechnol 41:42-52.

Walter M, Chaban C, Schutze K, Batistic O, Weckermann K, Nake C, Blazevic D, Grefen C, Schumacher K, Oecking C, et al. (2004) Visualization of protein interactions in living plant cells using bimolecular fluorescence complementation. Plant J 40:428-438.

Yamauchi T, Watanabe K, Fukazawa A, Mori H, Abe F, Kawaguchi K, Oyanagi A and Nakazono M (2014) Ethylene and reactive oxygen species are involved in root aerenchyma formation and adaptation of wheat seedlings to oxygendeficient conditions. J Exp Bot 65:261-273.

Yang YN, Li RG and Qi M (2000) In vivo analysis of plant promoters and transcription factors by agroinfiltration of tobacco leaves. Plant J 22:543-551.

Yang ZP, Li HL, Guo D, Tang X and Peng SQ (2014) Identification and characterization of the 14-3-3 gene family in Hevea brasiliensis. Plant Physiol Biochem 80:121-127.

Yoshie Y, Goto K, Takai R, Iwano M, Takayama S, Isogai A and Che FS (2005) Function of the rice gp91 ${ }^{\text {phox }}$ homologs OsrbohA and OsrbohE genes in ROS-dependent plant immune responses. Plant Biotechnol 22:127-135.

Zeng RZ, Duan CF, Li, XY, Tian WM and Nie ZY (2009) Vacuolar-type inorganic pyrophosphatase located on the rubber particle in the latex is an essential enzyme in regula- 
tion of the rubber biosynthesis in Hevea brasiliensis. Plant Sci 176:602-607.

Zhao XF, Colaizzo-Anas T, Nowak NJ, Shows TB, Elliott RW and Aplan PD (1998) The mammalian homologue of mago nashi encodes a serum-inducible protein. Genomics 47:319-322.

Zhao XF, Nowak NJ, Shows TB and Aplan PD (2000) MAGOH interacts with a novel RNA-binding protein. Genomics 63:145-148.

\section{Internet Resources}

Softberry platform for HMM-based gene structure prediction, http://linux1.softberry.com/berry.phtml

Multiple alignment software Clustal X, http://www.ebi.ac.uk/clustalw

MEGA5.2 software for phylogenetic tree construction, http://www.megasoftware.net/

GSDS software for comparison of cDNA sequences, http://gsds.cbi.pku.edu.cn/

Associate Editor: Marcia Pinheiro Margis

License information: This is an open-access article distributed under the terms of the Creative Commons Attribution License (type CC-BY), which permits unrestricted use, distribution and reproduction in any medium, provided the original article is properly cited. 